Revue

Revue de l'histoire des religions

de Ihistoire des religions

\title{
Les juifs et la nation au Moyen Âge
}

Jalons pour une étude

Jews and the Nation in the Middle Ages: Groundwork for a Study

Juliette Sibon et Claire Soussen

\section{(2) OpenEdition}

Édition électronique

URL : http://journals.openedition.org/rhr/8733

DOI : $10.4000 /$ rhr.8733

ISSN : 2105-2573

Éditeur

Armand Colin

Édition imprimée

Date de publication : 1 juin 2017

Pagination : 219-235

ISBN : 978-2-200-93126-1

ISSN : 0035-1423

Référence électronique

Juliette Sibon et Claire Soussen, « Les juifs et la nation au Moyen Âge », Revue de l'histoire des religions [En ligne], 2 | 2017, mis en ligne le 01 juin 2019, consulté le 06 janvier 2021. URL : http:// journals.openedition.org/rhr/8733; DOI : https://doi.org/10.4000/rhr.8733 
JULIETTE SIBON

Université d'Albi
CLAIRE SOUSSEN

Université de Cergy-Pontoise

\section{Les juifs et la nation au Moyen Âge Jalons pour une étude}

Si les médiévistes ont mis en évidence, depuis les travaux fondateurs de Colette Beaune, la pertinence du concept de nation pour définir les constructions étatiques de la fin du Moyen Âge, les études consacrées à une éventuelle "nation juive» médiévale ont longtemps fait défaut. Après avoir souligné les précautions méthodologiques nécessaires pour approcher ce sujet sensible, nous examinerons les sources latines et hébraïques, exégétiques et pratiques afin de recenser la terminologie qui pourrait la désigner à l'époque médiévale. Nous essaierons d'éclairer les sens revêtus par les termes de natio iudeorum ou gens iudeorum rencontrés dans les documents à la lumière des réflexions contemporaines sur le concept de nation au Moyen Âge, et surtout des sentiments exprimés par les médiévaux eux-mêmes.

\section{Jews and the Nation in the Middle Ages: Groundwork for a Study}

Starting with Colette Beaune's foundational works, Medievalists have demonstrated the pertinence of the concept of nation for understanding state-building in the late Middle Ages. However, studies focusing on a possible Medieval "Jewish nation" have long been lacking. After emphasizing the methodological precautions necessary for approaching this sensitive subject, we examine the sources, Latin and Hebrew, exegetical and practical, in order to identify the terminology that could express this notion in the Medieval period. We attempt to clarify the meanings of the terms natio iudeorum or gens iudeorum found in the documents based on contemporary thinking regarding nationhood in the Middle Ages, and especially based on sentiments expressed by Medieval people themselves. 
Les hérétiques nous harcèlent en faisant remarquer que Dieu a repoussé la fin de cet exil, plus durable que les autres. Mais ce n'est pas surprenant car Dieu ne punit pas une nation à moins que la mesure de ses péchés ait été pleine [...] Pourtant, la rédemption se traduira par la ruine, la destruction, le meurtre et l'éradication de toutes les nations, elles et les anges qui les protègent, ainsi que leurs dieux, comme il est écrit «Le Seigneur punira le résident des cieux dans les cieux et les rois de la terre sur la terre » [Isaïe 24, 21]. Jérémie aussi a dit «N'aie crainte Jacob, mon serviteur, dit le Seigneur, car je suis avec toi, j'anéantirai toutes les nations parmi lesquelles je t'ai dispersé, mais toi je ne t'anéantirai pas » [Jer. 46, 28]. Tu vois que Dieu détruira toutes les nations sauf Israël, comme Dieu nous l'a promis par Moïse ${ }^{1}$.

Cet extrait du Sefer Nizzahon yashan, ou Vieux livre de polémique, composé sans doute à la fin du XIII ${ }^{\mathrm{e}}$ siècle en terre ashkénaze, et constituant l'un des sommets de la littérature polémique antichrétienne médiévale, met en évidence plusieurs éléments clés pour l'historien en quête de la «nation » au Moyen Âge, et en particulier de la «nation juive ». Ceux-ci peuvent toutefois devenir des écueils faute de précautions méthodologiques et lexicales. Le terme «nation » est ici traduit (par nous à la suite de l'éditeur anglo-saxon) de l'hébreu Oumah (pl. Oumot) employé dans le texte par l'auteur anonyme de l'ouvrage. Oumah correspond assez précisément au terme «nation », même si son sens recouvre des réalités autres que celles qu'un auteur des périodes postérieures pourrait lui donner. La nation renvoie ici à Israël mais aussi aux « autres nations du monde» que les écrits juifs ou hébraïques qualifient plus souvent de goyim (peuples).

Pour éviter les contresens, les contextes interne et externe de l'œuvre doivent être précisés: le Sefer Niz̨ạhon yashan est un ouvrage de polémique et son auteur place Israël en situation de confrontation avec les autres peuples qui l'oppriment. Or le genre de la polémique donne souvent lieu à des affrontements réducteurs et manichéens, même si l'argumentation est fondamentalement subtile. En outre, dans ces textes, le thème de l'exil et des souffrances qui

1. Extrait du Nizzahon yashan, ou Vieux livre de polémique, édité dans The Jewish-Christian Debate in the High Middle Ages. A critical edition of the Nizzahon vetus with an introduction, translation and commentary by David Berger, Philadelphia, The Jewish Publication Society of America, 1979, p. 227. 
l'accompagnent renvoie en écho à celui de la terre d'élection qui vit s'épanouir une souveraineté désormais disparue. Enfin, le tournant du XIII ${ }^{\mathrm{e}}$ siècle, époque de la rédaction de l'œuvre, est caractérisée par un réel durcissement du discours des polémistes chrétiens sur les juifs d'Occident, et l'auteur du Sefer Nizzahon yashan exprime l'angoisse qui gagne de plus en plus ses contemporains. Est-ce à dire que les juifs médiévaux considéraient leur «peuple » comme une « nation », et la terre d'Israël comme leur « foyer »? On le voit, le risque d'anachronisme est grand et pour le déjouer, nous voulons embrasser un large éventail de sources, examinant les documents littéraires mais aussi les documents normatifs et ceux de la vie quotidienne. La confrontation des textes dits de «la pratique» - actes notariés et archives judiciaires latins, jurisprudence latine et rabbinique -, avec ceux émanant des savants, vise à examiner dans quelle mesure la «nation », notion si difficile à cerner au Moyen Âge, serait vectrice d'un processus de construction politique ou identitaire, d'un sentiment d'appartenance à une communauté qui se pense singulière mais fait partie d'un tout qui la transcende, ou au contraire, de la construction d'une communauté qui s'extrait ou qui subit le rejet.

\section{Historiographie et histoire de la nation au Moyen ÂGe}

Dans le champ des «études juives » médiévales, force est de constater que le sujet n'a jamais fait l'objet de travaux historiques comparables à ceux menés dans le champ de l'histoire politique et inaugurés en France de manière significative et incontournable par Colette Beaune ${ }^{2}$. Ainsi, Bernhard Blumenkranz, dont l'œuvre magistrale témoigne pourtant du souci constant de l'érudition, introduisait son article intitulé "Chemins d'un exil : 1306 », et publié pour la première fois en $1962^{3}$, par les considérations suivantes, qui apparaissent aujourd'hui quelque peu péremptoires :

Les changements intervenus dans l'histoire des Juifs en France, au moyen âge, peuvent être résumés par trois grands chapitres : 1/ Les

2. Colette Beaune, Naissance de la nation France, Paris, 1985.

3. Bernhard Blumenkranz, «Chemins d'un exil : $1306 »$, Évidences 92, 1962, p. 17-23, repris dans Bernhard Blumenkranz, Les Juifs en France. Écrits dispersés, Paris, Les Belles Lettres (« Franco-Judaïca », 13), 1989, p. 125-133. 
Juifs français, des origines de l'implantation jusqu'au $\mathrm{XI}^{\mathrm{e}}$ siècle ; 2/ Les Juifs en France, de la fin du XI ${ }^{\mathrm{e}}$ siècle jusqu'en 1306 ; 3/ Les Juifs de France, à partir de $1306^{4}$.

Blumenkranz considérait, en effet, l'année 1096 - terminus ad quem de son grand œuvre Juifs et chrétiens dans le monde occidental $^{5}$-, comme un tournant dans la condition des juifs en chrétienté. Selon lui, le haut Moyen Âge refusait «l'intolérance religieuse », tandis que l'aventure de la croisade constituait un facteur d'exclusion irréversible, dans la mesure où si les juifs «avaient été compagnons d'armes dans les combats de nation à nation », ils étaient nécessairement exclus de la guerre sainte. Et d'ajouter : «Bien plus grave encore : un véritable procès d'intention leur est fait [...] : ils pencheraient du côté des musulmans s'ils avaient la possibilité de se prononcer. Ils sont considérés ainsi comme alliés en puissance de l'ennemi extérieur $»^{6}$.

Quant à Simon Schwarzfuchs, il n'envisage même pas la question dans l'ouvrage qu'il consacre en 1986 à la communauté juive de l'Europe médiévale :

Il n'y eut donc pas de communautés nationales juives dans l'Europe médiévale. Il est vrai que le concept de nation lui-même n'avait pas encore réussi à s'imposer. [...] L'horizon des communautés médiévales ne dépassa pas le plan local, ou celui de la seigneurie dont elles dépendaient, même quand les rois ou les empereurs considéraient que tous les Juifs des pays placés sous leur domination dépendaient d'eux et leur devaient l'impôt. Ils durent se plier à l'occasion, payer leurs impôts en commun et obéir aux dirigeants qui leur furent donnés : ils ne renoncèrent pas pour autant à la volonté d'autonomie de leurs communautés ${ }^{7}$.

Les hommages récents organisés à l'occasion du centenaire de la naissance de Blumenkranz en 2013 ont été l'occasion de souligner l'ampleur de l'héritage qu'il a légué aux historiens des juifs médiévaux ${ }^{8}$. Maître au sens artisanal du terme, « historien français

4. Ibid., p. 125.

5. Bernard Blumenkranz, Juifs et chrétiens dans le monde occidental, 430-1096, Peeters, Paris-Louvain, 1960, rééd. 2006.

6. Bernard Blumenkranz, « Chemins d'un exil : 1306 », art. cit., p. 125.

7. Simon Schwarzfuchs, Kahal. La communauté juive de l'Europe médiévale, Paris, Maisonneuve et Larose, 1986, p. 27.

8. Claude Denjean et Juliette Sibon, « Être historien des juifs médiévaux en France après Bernhard Blumenkranz », Philippe Buc, Martha Keil et John Tolan dir., Jews ans Christians in Medieval Europe : The Historiographical Legacy of Bernhard Blumenkranz, Turnhout, Brepols, p. 349-365. 
de langue française » comme il se présentait lui-même, promoteur de la transdisciplinarité et de la transpériodicité, du dialogue entre les sources de toute nature, juives et non-juives, écrites et non-écrites, chantre de l'étude lexicale, il invitait les historiens à renouveler les réponses aux anciennes questions, à faire émerger des problématiques novatrices, à se départir des anachronismes et des généralisations, à exhumer des documents inédits. Introduire ainsi notre réflexion sur «Les juifs et la nation au Moyen Âge» ne peut que nous inciter à soumettre le propos au crible exclusif de la démarche historique, à faire nôtres les mises en garde méthodologiques préliminaires à cette question, jamais prise en compte de manière aussi explicite que nous proposons de le faire. En dépit du soupçon d'instrumentalisation dont elle fait parfois l'objet - non sans raison d'ailleurs - de participation à l'écriture de tel ou tel «roman national», l'étude de la «nation» dans le contexte juif médiéval mérite que nous nous y penchions, non pour servir une entreprise téléologique dont l'objectif serait d'exhumer dans des siècles anciens les justifications d'idéologies du présent, mais pour faire œuvre d'historien, au sens où Blumenkranz - pour ne citer que lui et puisque nous réfléchissons dans le champ des « études juives »-, l'entendait.

« Un tel sujet ne s'inscrit ni hors du temps ni hors du sol», soulignait Pierre Monnet dans l'introduction au colloque que la Société des Historiens Médiévistes de l'Enseignement Supérieur Public, a consacré, à Prague, au thème "Nation et nations au Moyen Âge » en juin 2013 . L'historien d'aujourd'hui pense et écrit dans un monde globalisé, animé par le réveil des frontières, à l'origine de replis sur soi, de tentations «nationalistes », quelle qu'en soit l'acception. En France, il doit composer avec la concurrence des mémoires d'une part, qui renvoie à la multiplication des revendications mémorielles et à l'inflation des cérémonies publiques de commémoration depuis quelques décennies, et avec celle de l'histoire et de la mémoire d'autre part, non sans ignorer, qui plus est, les difficultés que connaît l'enseignement au collège et au lycée en général, et celui de l'histoire en particulier. Pour autant, la pensée

9. Pierre Monnet, « Nation et Nations au Moyen Âge : introductions », Société des Historiens Médiévistes de l'Enseignement Supérieur Public, Nation et Nations au Moyen Âge, Paris, Publications de la Sorbonne, 2014, p. 9-34, ici p. 17. 
méthodique peut s'exercer sur la «nation » comme sur tout autre objet.

D'ailleurs, si la cliométrie peut être trompeuse, car les titres d'ouvrages ne mettent pas souvent en exergue le mot «nation », les médiévistes ne délaissent pas la question, loin de là. À la croisée de l'histoire politique ou « histoire des pouvoirs publics » comme elle est parfois revendiquée pour le Moyen Âge, de l'histoire culturelle et de la plus récente histoire des «identités» ou des « appartenances », sous-champs académiques commodes bien que réducteurs, l'historiographie de la nation est plus foisonnante qu'il y parait, y compris depuis la déconstruction du mythe de « la France éternelle» par les historiens. Elle s'est effectivement renouvelée, loin des « histoires serves », telles que les qualifiait Lucien Febvre, destinées à nourrir les nationalismes. Entre l'affirmation que les nations contemporaines ont hérité du sentiment national qui se serait développé au Moyen Âge, et celle qui prétend que la nation est fille de la Révolution française, le médiéviste s'inscrit en faux, à l'instar de Jean-Marie Moeglin, qui envisage, pour simplifier, des nations médiévales sans nationalismes ${ }^{10}$.

Encore faut-il comprendre les acceptions médiévales du terme «nation». Dans son Apologie pour l'Histoire publiée à titre posthume pour la première fois en 1949, Marc Bloch invitait ainsi l'historien «à la poursuite de l'erreur et du mensonge»: «Au grand désespoir des historiens, les hommes n'ont pas coutume de changer de vocabulaire chaque fois qu'ils changent de mœurs $»^{11}$. Le terme de «nation", du latin nasci, «naître », qui désigne au Moyen Âge des groupements très divers - à l'instar des «nations » universitaires $^{12}-$, ne connaît de sens politique qu'au $\mathrm{XV}^{\mathrm{e}}$ siècle - et se décline alors au singulier, pour désigner les habitants d'un pays ou les membres d'une communauté pourvue d'une identité fondée sur l'origine. Ce terme constitue l'un de ces "guêpiers sémantiques ", pour reprendre la formule de Colette Beaune ${ }^{13}$, dont l'historien doit

10. Jean-Marie Moeglin, «Nation et nationalisme du Moyen Âge à l'époque moderne (France-Allemagne) », Revue historique 123/3 (1999), p. 537-554.

11. Marc Bloch, Apologie pour l'histoire ou Métier de l'historien, Paris, [1949], rééd. 1997, p. 57.

12. Voir notamment Nathalie Gorochov, « Genèse et organisation des nations universitaires en Europe », Nation et Nations..., op. cit., p. 273-286.

13. Colette Beaune, «Nation», Claude Gauvard, Alain de Libera et Michel Zink dir., Dictionnaire du Moyen Âge, Paris, Puf, 2002, p. 986-987. 
se départir, afin de «distinguer ce que le vulgaire confond», et de ne pas opérer de «conceptualisation sauvage », par simple transfert, en plaquant les réalités du présent sur celles du passé ${ }^{14}$. Ainsi, la « nation » peut être pensée bien en amont de la Révolution française.

Avec Naissance de la nation France, publié en 1985, Colette Beaune a incontestablement renouvelé l'histoire politique de la fin du Moyen Âge en abordant l'analyse de la nation dans la France des $\mathrm{XIV}^{\mathrm{e}}$ et $\mathrm{XV}^{\mathrm{e}}$ siècles, à travers l'imaginaire. L'imaginaire de la nation tel que les écrits savants l'élaborèrent, ne saurait être coupé de ses racines religieuses : c'est un imaginaire chrétien. Les mythes d'origine, ceux des Troyens et de Clovis, et les saints intercesseurs de la nation auprès de Dieu, à savoir saint Denis - le martyr mais aussi le lieu -, Saint Louis et saint Michel, contribuent à faire de la nation une valeur, certes, mais une valeur parmi d'autres, transcendée par l'amour de Dieu et l'amour du prochain.

Toutefois, les universitaires de la fin du Moyen Âge n'exclurent pas totalement la conception aristotélicienne fondée sur la notion de « bien commun ». D'ailleurs, à partir du tournant du XIVe siècle, la «patrie», oubliée depuis la fin de l'Antiquité car perçue jusque-là comme indissociable du paganisme, connut une forme de christianisation. Remise au goût du jour, elle n'était pas de ce bas monde : c'était la Jérusalem céleste, celle pour qui l'on pouvait mourir en martyr. Mais la nouvelle pensée universitaire, qui s'appuyait sur Aristote, enrichit l'acception. L'amour de la patrie transcendait désormais le cadre familial et était associé au courage et à la défense de la liberté et des lois. Il devenait magnanime de mourir pour cette patrie-là, non céleste ${ }^{15}$. Certes, l'idée que l'on pût et dût se sacrifier pour la patrie-royaume d'ici-bas, d'abord promue par Philippe Auguste, puis reprise avec force par les propagandistes de Philippe le Bel, ne s'imposa que tardivement, au début du $\mathrm{XV}^{\mathrm{e}}$ siècle, en pleine guerre de Cent Ans, après le choc de l'invasion de 1415-1416, et chez les Armagnac d'abord ${ }^{16}$. Mais, les derniers Capétiens promurent un autre patriotisme profane, à travers la contribution au fisc royal ${ }^{17}$.

14. Joseph Morsel, « De l'usage des concepts en Histoire médiévale », Ménestrel, Médiévistes sur le net: sources, travaux et références en ligne, www.menestrel.fr.

15. Colette Beaune, Naissance de la nation France..., op. cit., p. 439-444.

16. Ibid., p. 448.

17. Ibid., p. 437-439. 
Or, les juifs de leur royaume apparaissaient, d'une manière ou d'une autre, comme des acteurs du patriotisme fiscal. En les revendiquant pour la première fois comme Iudei nostri - « nos juifs »-, Louis VIII les intégrait au Trésor royal. Louis IX le confirmait par sa législation. Il dit même à Guillaume de Chartres, son historiographe officiel à partir de 1248 : «Que les évêques fassent ce qui les regarde au sujet des chrétiens qui dépendent d'eux. Quant à moi, je veux faire ce qui m'appartient au sujet des juifs $»^{18}$.

Alors pourquoi chercher du côté de la nation un supplément de sens pour définir l'entité juive médiévale? La réponse est qu'en dépit des réserves épistémologiques que nous avons soulignées, la quête semble a priori pertinente pour les deux acceptions du terme nation : celle de la natio au sens médiéval premier du terme, c'està-dire de groupe identitaire au sein d'une entité plus large, comme l'étaient les nations universitaires par exemple; celle, que l'on s'autorise à considérer comme hypothèse de travail, de «nation » au sens contemporain du terme, désignant un corps de dimension supranationale ou extraterritoriale dont les membres sont liés par des références identitaires communes. Il faut donc définir ou identifier une «nation juive » différente des nations médiévales émergentes telles que les historiens les analysent, produits d'un paradigme associant les hommes, le sentiment ou la volonté et le territoire, et qui toutes, apparaissent rassemblées et localisées.

\section{NATURE ET LIMITES}

\section{DES ENTITÉS POLITIQUeS JUIVES AU MoYen ÂGE}

Dans le contexte juif médiéval, en effet, le terme de «nation» apparaît dans les écrits hébraïques, latins et de langue vulgaire, complété par un répertoire de mots connexes que l'historien intègre à son corpus. Par exemple, les notaires latins provençaux mobilisent la formule de natio judeorum de manière exceptionnelle - dans un contexte exclusivement fiscal, semble-t-il - et usent, en revanche, régulièrement des termes de communitas ou universitas

18. Guillaume de Chartres, De Vita et de Miraculis, p. 34, cité et traduit dans Jacques Le Goff, Saint Louis, Paris, Gallimard, 1996, p. 795. 
judeorum. L'exégèse biblique latine, quant à elle, mobilise d'autres termes sur lesquels nous allons revenir. Les deux documentations font d'ailleurs un usage différencié des termes hebreus et judeus, le premier toujours connoté positivement, qui renvoie à la langue et à la culture, le second soit péjoratif, pour désigner les juifs contemporains, soit d'un emploi neutre strictement juridique ${ }^{19}$.

Bref, chaque type de sources et chaque langue fournissent les bases d'études sémantiques préalables à toute analyse. On peut aussi inclure le vocabulaire iconographique, celui des enluminures, à l'instar de celle que nous avons choisie pour illustration en quatrième de couverture, «Moïse et la sortie d'Égypte », tirée d'une Bible latine datée du XIV $^{\mathrm{e}}$ siècle et conservée à la Bibliothèque municipale de Tours $^{20}$, des vitraux mais aussi des dessins qui illustrent les Statuts municipaux des villes du Midi de la France ou les fueros ibériques, et les confronter à nos textes.

Cependant, si les historiens médiévistes pensent la «nation», ils persistent à considérer que le cas des juifs reste «à part». Les œuvres collectives - dont, parmi les plus marquantes pour la France, celles rassemblées dans les tomes II et III des Lieux de Mémoires coordonnés par Pierre Nora, la Nation paru en 1986 et Les France paru en 1992, auxquels ont participé les grands spécialistes de l'époque, telle Colette Beaune, ou encore Bernard Guenée, Georges Duby, Jacques Le Goff et Philippe Contamine notamment - ignorent le sujet ${ }^{21}$. Ou encore, très récemment, le volume paru en 2014 sous la direction de Mikhaïl Dmitriev et Daniel Tollet, intitulé Confessiones et nationes. Discours identitaires et nationaux dans les cultures chrétiennes, propose, pour le Moyen Âge et l'époque moderne, trois parties consacrées au catholicisme, au protestantisme et à l'orthodoxie ${ }^{22}$. Quant aux index thématiques des tables de la Revue des Études Juives, par exemple, ils sont dépourvus d'entrée «nation » pour la période médiévale.

19. Claude Denjean, Juliette Sibon et Claire Soussen, « La nation juive à la fin du Moyen Âge. Mythe ou réalité ? Fantasme ou utopie ? », dans Nation et Nations au Moyen Âge, op. cit., p. 287-298.

20. Tours - BM - ms. 0008, fol. 24.

21. Pierre Nora dir., Les Lieux de Mémoire, La République. La Nation. Les France, vol. 1, rééd. Paris, Gallimard, 1997.

22. Daniel Tollet et Mikhaïl Dmitriev, Confessiones et nationes. Discours identitaires nationaux dans les cultures chrétiennes : Moyen Âge $-X^{e}$ s., Paris, Honoré Champion, 2014. 
Finalement, ce sont surtout les études développées à l'échelle de la ville, celle des cités de l'Europe méditerranéenne en particulier, qui offrent la citoyenneté à leurs habitants, chrétiens comme juifs, qui abordent indirectement le questionnement sur la «nation des juifs », la «nation juive » et « les juifs dans la nation » au Moyen Âge. L'histoire urbaine, en effet, à l'aune des problématiques sur les mobilités, l'étranger, la marginalité et les minorités, développe la réflexion par le biais des questions de l'insertion et de la convivencia ou « vivre ensemble».

Citons, sans exhaustivité, les travaux d'Henri Bresc sur la Sicile médiévale, de Claude Denjean sur la Couronne d'Aragon, ou encore de Noël Coulet sur la Provence angevine ${ }^{23}$. L'intégration des juifs à la civitas, par le biais de la propriété foncière, de la détention d'offices publics en dépit de l'interdiction canonique, de la participation à la défense de la cité et de la reconnaissance de la vertu citoyenne, se lit notamment dans la construction d'une communis opinio, de valeurs urbaines partagées entre chrétiens et juifs, dont, en positif, l'attachement à la cité d'origine ${ }^{24}$. Parmi les plus beaux témoignages de l'amour de la petite patrie, la fameuse stèle d'Olot - dans la province de Gérone - garde gravé à jamais le souvenir ébloui que les juifs originaires de Béziers, temporairement réfugiés en Catalogne, ont conservé de leur ville après l'assaut des troupes de Simon de Montfort en $1209^{25}$. Ces valeurs urbaines interconfessionnelles recèlent aussi un versant négatif, la xénophobie, y compris contre des coreligionnaires originaires de cités «étrangères ». En effet, à une supposée solidarité intercommunautaire fondée sur la pratique religieuse, la langue voire l'origine commune, répondait une sociabilité interreligieuse clai-

23. Henri Bresc, Arabes de langue, juifs de religion. L'évolution du judaïsme sicilien dans l'environnement latin, XII -XV siècles, Paris, Bouchène, 2001 ; Claude Denjean, La loi du lucre. L'usure en procès dans la Couronne d'Aragon à la fin du Moyen Âge, Madrid, Casa de Velázquez, 2011 ; Noël Coulet, «Les juifs en Provence au bas Moyen Âge : les limites d'une marginalité », Pierre Tucoo-Chala et Béatrice Leroy dir., Minorités et marginaux en Espagne et dans le midi de la France (VII -XII ${ }^{e}$ S.), Paris, CNRS, 1986, p. 203-216.

24. Claude Denjean et Juliette Sibon, «Citoyenneté et fait minoritaire dans la ville. Étude comparée des juifs de Marseille et de la Couronne d'Aragon au bas Moyen Âge », Revue d'Histoire urbaine 32 (2011), p. 73-100.

25. Gérard Nahon, Inscriptions hébraïques et juive de France médiévale, Paris, Franco-Judaica, 1986, p. 342-345. 
rement visible dans les sources dites « de la pratique », qui distinguaient le citoyen juif du judeus extraneus.

La vie politique qui animait les communautés juives dans les derniers siècles du Moyen Âge est un autre développement de la réflexion. Par exemple, les responsa ou consultations rabbiniques de Salomon ben Adret à Barcelone dans la seconde moitié $\mathrm{du} \mathrm{XIII}^{\mathrm{e}}$ siècle révèlent les tensions politiques au sein de la communauté ou qahal en hébreu. Les conflits portaient sur les finances et la fiscalité. Les sources sont rares mais on les observe clairement à Aix-en-Provence au $\mathrm{XV}^{\mathrm{e}}$ siècle, ainsi que dans les communautés siciliennes aux $\mathrm{XIV}^{\mathrm{e}}$ et $\mathrm{XV}^{\mathrm{e}}$ siècles en particulier, au sein desquelles Henri Bresc mit au jour «l'oligarchie rampante» et les querelles partisanes qui clivaient les assemblées des communautés ${ }^{26}$.

L'intense vie politique interne aux communautés juives, clairement observée dans l'Occident méditerranéen, pose enfin la question de l'autonomie des juifs. «Petit état intra-territorial, presque totalitaire bien qu'apolitique » pour Salo Baron, dans un contexte de disparition des liens féodaux et d'essor des corporations ${ }^{27}$, les communautés juives médiévales n'avaient, au contraire, pour Maurice Kriegel, aucune autonomie dans la mesure où les fédérations intercommunautaires servaient uniquement les intérêts de l'autorité non juive en facilitant la collecte des impôts ${ }^{28}$.

Cette question de l'existence ou non d'un embryon d'État communautaire peut assurément être éclairée par l'appréhension du sentiment des juifs médiévaux, d'un sentiment «national»

26. Danièle Iancu-Agou, «La communauté juive aixoise à l'extrême fin du $\mathrm{XV}^{\mathrm{e}}$ siècle : dissensions internes et clivage social », Proceedings of the Seventh World Congress of Jewish Studies, Jérusalem, World Union of Jewish Studies, 1981, p. 9-27 ; Henri Bresc, « Gli ebrei di Sicilia e la politica nel Medioevo », Siculorum gymnasium, 56-2, 2003, p. 263-284, et «La démocratie dans la communauté et dans l'État en France méridionale et en Provence aux derniers siècles du Moyen Âge », Actes de l'Université Occitane d'été 2002-2003, Jòrgi Peladan dir., Nîmes, Marpoc, 2005, p. 21-36 ; Juliette Sibon, «Les juifs et la contestation politique au bas Moyen Âge. Midi de la France, Couronne d'Aragon, Sicile », Gilles Lecuppre dir., La contestation (Moyen Âge et Temps modernes), Paris, Éditions Kimé, 2016, p. 135-158.

27. Salo Baron, The Jewish Community. Its History and Structure to the American Révolution, Philadephie, Greenwood Press, 1962, vol. 1, p. 208.

28. Maurice Kriegel, Les juifs à la fin du Moyen Âge dans l'Europe méditerranéenne, Paris, Hachette, 1979, p. 111-112. 
avec tous les guillemets qui s'imposent. En effet, si tant est que l'on admette l'existence d'une nation juive médiévale, celle-ci est-elle pensée ou conscientisée par ses membres ? Existe-t-il un sentiment identitaire et comment l'appréhender? Et s'il existe, peut-on l'assimiler au sentiment national ou proto-national ?

Le point de départ de la réflexion réside dans le constat qu'il existe depuis les exils babylonien et romain ${ }^{29}$, des communautés juives dispersées et caractérisées par un certain nombre de points communs qui font qu'elles sont assimilables entre elles ou les unes aux autres, et inassimilables dans les terres qui les accueillent. Par ailleurs, signe de leur parenté durable, ces communautés gardent des liens entre elles et avec leur foyer primitif, la Terre Sainte.

Ce que l'on peut commencer par qualifier de «personnalité » ou d' «identité » juive, même si le terme fait débat, se caractérise par des facteurs distinctifs au sein de leurs terres d'accueil. La présence juive est en effet relativement uniforme où qu'on l'observe, caractérisée par des infrastructures, des modalités d'organisation et des institutions de gestion interne souvent similaires. Par ailleurs, la langue qu'emploient les juifs où qu'ils soient pour leur culte est la même, l'hébreu, et leurs références juridiques sont elles aussi uniformes, tirées de la Torah et du Talmud.

C'est bien ce que défendait Benjamin de Tudèle, dans son Sefer massa'ot ou Livre des voyages écrit dans le dernier quart $\mathrm{du} \mathrm{XII}^{\mathrm{e}}$ siècle, en dressant « un état moral et religieux de ses frères dispersés $»^{30}$. Il relata son itinéraire autour du bassin méditerranéen au travers d'une succession de notices brèves et répétitives, consacrées à des communautés juives (Gérone, Narbonne, Lunel, Marseille...), en précisant leur taille, les trois ou quatre personnalités réputées pour leur connaissance de la Torah et de la Halakha, et, le cas échéant, la présence d'une yeshiva. Son récit, qui connut tant de succès dès le Moyen Âge et dont la postérité n'est pas à démontrer, livre une récapitulation de l'étendue du monde juif, un tableau de la diaspora, de ce qui faisait alors l'unité et la pérennité du judaïsme, à savoir la référence à un calendrier commun, l'usage

29. Simon Mimouni emploie pour désigner les habitants de ce foyer primitif le terme de «nation judéenne ». Voir aussi Shamai Gelander, From Two Kingdoms to One Nation - Israel and Judah, Brill, Leiden-Boston, 2011.

30. Eliakim Carmoly, Notice historique sur Benjamin de Tudèle, Kiessling \& C $\mathrm{C}^{\mathrm{ie}}$, Bruxelles et Leipzig, 1852, p. 9. 
de l'hébreu pour la liturgie et les savoirs, l'étude de la Torah et du Talmud ${ }^{31}$.

Quant aux sources littéraires qui évoquent la douleur de l'exil, elles appréhendent la «nation» de manière indirecte, par la référence à la Terre Sainte objet de regrets. Le registre de la déploration employé dans les chroniques relatives au temps des croisades par les juifs du Rhin, mais aussi dans les commentaires bibliques des sages de Séfarad des $\mathrm{XII}^{\mathrm{e}}$ et $\mathrm{XIII}^{\mathrm{e}}$ siècles, tel David Kimhi ou Rabbi Meïr de Narbonne, oppose systématiquement une personnalité juive collective éprouvant les rigueurs de l'exil, à une majorité dominante et exclusive. C'est donc bien une nation sans État, et évidemment sans terre qui est définie en creux par le répertoire hébraïque, et là encore de façon négative. La recherche gagnera beaucoup à sonder le terrain de la philologie latine et hébraïque afin de mettre en évidence les relations intertextuelles que nous devinons sans qu'elles s'expriment ouvertement. D'après l'historien Yehuda Shamir ${ }^{32}$, Juda Ha-Levi lorsqu'il écrivit son Kuzari avait à l'esprit la souffrance des juifs (d'Israël) et il développa la théorie selon laquelle parce qu'Israël est la nation choisie par Dieu pour jouer un rôle vital parmi les nations, elle souffre plus que les autres.

Mais pour ajouter un supplément d'âme à ce que serait la nation juive médiévale, peut-on la définir de façon positive ? Observet-on au Moyen Âge et de façon sans doute anachronique, voire hérétique pour la démarche historique, cette nation «plébiscite de tous les jours » que définit Ernest Renan ${ }^{33}$ ? Les juifs médiévaux se pensent-ils comme un tout immanent et pérenne, ou même comme une entité politique spécifique ? On serait tenté de répondre par l'affirmative lorsqu' on étudie les démarches menées par les chefs de communautés locales ou les halakhistes pour

31. Juliette Sibon, «Benjamin de Tudèle, géographe ou voyageur? Pistes de relecture du Sefer massa'ot », Henri Bresc et Emmanuelle Tixier du Mesnil dir., Géographes et voyageurs au Moyen Âge, Nanterre, Presses universitaires de Paris Ouest, 2010, p. 207-223.

32. Yehuda Shamir, Rabbi Moses Ha-Kohen of Tordesillas and his Book Ezer Ha-Emunah, Leiden, Brill, 1975, p. 42.

33. Ernest Renan, Qu'est-ce qu'une nation ?, réédition, Paris, 2011. Schlomo Sand qui présente le texte de Renan explique que : « Aux yeux de Renan, la nation est avant tout une solidarité entre des sujets modernes et autonomes, désireux de vivre sous une même souveraineté ». Ce n'est pas le cas des juifs médiévaux évidemment. 
faire reconnaître les prérogatives qui leur sont accordées dans certains territoires, ou les privilèges qui reconnaissent l'existence d'une personnalité juridique juive dans le cadre des communautés locales. Mais dans ces cas-là, ces petites entités autonomes et spécifiques s'apparentent davantage aux nationes locales qu'évoque la documentation de la pratique, communitates ou universitates judeorum bénéficiaires de privilèges particuliers au sein des États dans lesquels elles s'inscrivent. Elles obtiennent la reconnaissance par les autorités des États dans lesquels elles existent, d'institutions qui les distinguent et leur assurent une certaine autonomie. Louis Finkelstein était même allé jusqu'à parler de Jewish selfgovernment pour désigner cette autonomie ${ }^{34}$.

En revanche, si l'on retient comme valide l'hypothèse de petites nations juives sans État au Moyen Âge, ce qui est une évidence, la nation juive englobante reste un fantôme. En effet, au Moyen Âge - faut-il le préciser ? -, il n'y a pas de revendication nationale juive au sens positif du terme. On trouve cependant les indices de l'existence de la nation juive médiévale sur les terrains juridiques et politiques. Les Sages du Talmud - dont l'importance ne fait que croître tout au long du Moyen Âge en Occident - formalisent dès le III $^{\mathrm{e}}$ s. le principe Dina de Malkhuta Dina, la Loi de l'État est la Loi. Ce principe reflète bien l'idée qu'il existe des États/royaumes au sein desquels vivent les juifs et auxquels ils sont soumis en tant que groupe particulier uni par d'autres règles de portée interne. Il y a donc emboîtement d'une entité juive - peut-on l'appeler «nation»? - au sein de chaque État qui l'accueille. Il faut, dans le même temps, souligner le paradoxe qui fait que par cette double sujétion au prince du pays d'accueil et à Dieu, les juifs sont par essence inassimilables et perdurent. Par ailleurs à partir du début du XIII $^{\mathrm{e}}$ siècle, à travers la législation du concile de Latran IV (1215), la logique qui prévaut en chrétienté est de mieux les distinguer pour les tenir à distance, identifier et marquer les individus mais aussi les groupes, ce qui tend à favoriser les particularismes. Cette nation juive sans État, réalité immatérielle, est d'ailleurs consciemment assumée par les juifs médiévaux qui interprètent la dispersion comme le juste châtiment des péchés commis envers

34. Louis Finkelstein, Jewish Self-Government in the Middle Ages, New-York, Greenwood Press, 1925. 
Dieu. Il y a donc ambivalence entre la déploration récurrente quant au sort vécu en exil et un destin politique parfaitement assumé. Pourtant, on l'a dit, institutionnellement et légalement, les juifs font tout pour se voir garantir leur exceptionnalité.

Pour finir, et pour sortir des hypothèses, il convient de revenir sur les mots. C'est en réalité dans l'exégèse qu'on rencontre le terme de «nation » le plus directement et le plus sûrement appliqué à l'entité juive, au travers des termes populus, gens et stirps iudeorum, que les spécialistes s'autorisent à traduire dans certains contextes exégétiques par «nation juive». L'expression est alors utilisée pour désigner la globalité que constitue l'autre, le minoritaire, celui qui a refusé de reconnaître la Vérité du christianisme. Pour l'exégète ou le polémiste, les juifs se caractérisent avant tout par leur infidélité à l'égard de l'alliance divine. L'exégète ou le théologien n'a que faire des considérations étatiques ou politiques. Son analyse est macro-scalaire, et dans sa conception, les juifs ne constituent qu'un groupe, une nation parmi d'autres, là où la chrétienté à vocation à rassembler toute l'humanité sans distinction.

Dans son Adversus Iudaeos, Tertullien, qui commente Gn. 25-23 à la fin du $\mathrm{II}^{\mathrm{e}}$ siècle, reprend les termes utilisés par les Pères de l'Église, à savoir populus et gens, qui désignent plus tard la nation. À la fin du IV ${ }^{\mathrm{e}}$ siècle, saint Augustin est l'un des premiers qui utilise le terme stirps pour désigner les juifs dans sa Cité de Dieu, ouvrage qui définit de manière précise et presque programmatique la position des juifs en chrétienté ${ }^{35}$. Les juifs sont pris dans leur globalité comme «peuple témoin »-comme il existe des buttestémoins en géologie. Ils sont les garants, à leur corps défendant, de la véridicité de la Passion du Christ et, en tant que tels, indispensables. Deux siècles plus tard, Isidore de Séville emploie les mêmes termes, enfermant les juifs dans une communauté constituée autour de l'infidélité. Il s'agit donc bien d'une nation en négatif. Au XIII ${ }^{\mathrm{e}}$ siècle, Pierre de Jean Olieu utilise lui aussi gens pour désigner les juifs au même titre que les autres peuples

35. Saint Augustin, De civitate Dei, Livre 20 chapitre 30 : «Sed sicut dicimus Iudaeis: "Vos occidistis Christum", quamuis hoc parentes eorum fecerint: sic et isti se dolebunt fecisse quodam modo, quod fecerunt illi, ex quorum stirpe descendunt»; «Numquid nisi Dei est auferre omnes gentes inimicas sanctae ciuitatis Hierusalem ». 
païens ${ }^{36}$, de même qu'Alphonse Bonhomme au début du XIV siècle les assimile aux peuples qui se convertiront ${ }^{37}$. Déjà, au début du $\mathrm{XII}^{\mathrm{e}}$ siècle, Petrus Alfonsi dans son Dialogue contre les juifs faisait employer l'expression par son ancien moi, Moïse, qui accusait son nouveau moi, Pierre, d'opprimere gentem Judaicam ${ }^{38}$. Là encore les juifs sont enserrés dans une collectivité négative.

Quant au registre profane des textes juridiques ibériques de la fin du Moyen Âge, il atteste la réactivation du concept de stirps que l'on peut traduire dans ce contexte par « race» ou «souche». Lorsque les conversions se multiplient dans le second tiers du $\mathrm{XV}^{\mathrm{e}}$ siècle, le terme réapparaît à Tolède et en Castille. La SentenciaEstatuto de Pedro Sarmiento mobilise explicitement le «lignage» et la « race $»^{39}$. L'auteur accuse les «conversos del linage [sic] de los judios", les «descendientes del linaje [sic] de los judios» et les «descendientes del linaje y ralea de los judios », d'être les ennemis des $«$ vecinos christianos viejos $»^{40}$.

Dans le comté de Provence, enfin, sous domination angevine depuis le milieu du XIII ${ }^{\mathrm{e}}$ siècle, c'est bien le terme de natio qui est employé au lendemain des massacres de 1348 par les juifs qui négocient le montant de l'impôt annuel, ou tallia judeorum, avec les représentants du pouvoir comtal. Le texte, issu du célèbre registre de la taille des juifs de Provence et daté du 26 juillet 1348, relate des massacres qui auraient abouti à réduire la « nation » juive à néant ou presque (ipsorum natio est quasi reducta ad nichilum) ${ }^{41}$.

36. Pierre de Jean Olieu, Lectura super Genesim, BnF Ms lat 15559, f $96 \mathrm{ra}$ : «Idcirco omnes eos redire ad terram patrum suorum ubi et in fine ad iudeos convertendos redibunt et sic apostoli relicta synagoga fugerunt ad gentes ...».

37. Alphonse Bonhomme, Epistola Raby Samuel Marochanus... PL 149, Cap. XVI, col. 353 : « Dixit adhuc idem propheta de illis : "Ecce gentem quam nesciebas vocabis et nationes quae te non cognoverunt ad te venient (Isa. Liii)". Et sicut de facto videmus hodie sunt mille anni et ultra quia Christus missus secundum legem nobis datam venit, et gentes quae legem non noverant, venerunt ad eum et ipse dedit eis legem novam, puram, sanctam ».

38. Petrus Alfonsi, Dialogi adversus iudaeos, PL 157, fin du prologue, col. 540 : «Nimis profunde in nostram inveheris infamiam, et vis opprimere gentem Judaicam $»$.

39. Antonio Martín Gamero, Historia de la ciudad de Toledo: sus claros varones y monumentos, Toledo, Imprenta de Severiano López Fando, 1862, p. 1036-1040.

40. Id., ibid., p. 1039.

41. Archives départementales des Bouches-du-Rhône B 2564, dans Joseph Shatzmiller, «Les juifs de Provence pendant la Peste noire », Revue des études juives CXXXIII (1974), p. 457-480. 
En définitive, tous ces travaux, tant ceux des historiens médiévistes, spécialistes ou non des études juives, que ceux des philosophes et des philologues, terreaux de nos questionnements, attestent combien le Moyen Âge est riche de perspectives pour penser le «national » et «l'universel». Force est de constater que chez les auteurs médiévaux, chrétiens comme juifs, l'ambivalence domine quant à l'existence et à la revendication d'une «nation juive » ou « des juifs ». Il y a bien une «nation juive », ancienne, immuable. Il y a aussi une «nation des juifs », vécue au quotidien, en devenir, mais dont les contours sont flous et toujours labiles car, en effet, la vigueur des particularismes culturels, les divergences religieuses entre judaïsme rabbinique et karaïte, le rapport à la Terre sainte et la question du messianisme font émerger des lignes de clivage qui ne militent pas en faveur d'une nation transcendantale universellement promue au sein du judaïsme.

sibonjuliette@aol.fr

claire.soussen@wanadoo.fr 